COMMENT. This study shows that speech manifestations are common in complex partial seizures of temporal lobe origin and can help to lateralize the origin of the seizure. John Hughlings Jackson noted that seizures in the dominant hemisphere could result in speech abnormalities and Penfield found that electrical stimulation of the speech areas in the dominant hemisphere produced dysphasia whereas stimulation of the motor speech area of either hemisphere produced vocalization.

\title{
BODER'S SUBTYPES OF DYSLEXIA
}

The subtyping of dyslexic children proposed by Boder $(1971,1973)$ have been validated by quantitative neurophysiological techniques at the Gunderson Medical Foundation, LaCrosse, WI. Children were classified as being dysphonetic (auditory-phonetic disabilities), dyseidetic (visual spatial disabilities) or mixed (deficient in both processes). In one study of 21 dyslexic children between 7 and 10 years of age and six controlled children, there were significant differences between the dyslexic subgroups and between the dyslexic and controlled children on three of the six cognitive tasks (frustration level reading, spelling recognition, and drawing a clock). Significant differences occurred in left temporal parietal theta, and this difference occurred in the area of the angular gyrus, presumed to be important in phonetic decoding. This suggested that the reportedly normal phonetic skills of dyseidetic children may not be normal but rather a sign of overuse of a processing strategy associated with inefficiency of right hemisphere visual gestalt abilities. Additional support for an overuse theory in dyseidetic dyslexia came fram the behavior of the children during reading tasks. The dyseidetic children audibly decoded many words whereas dysphonetic and mixed dyslexics skipped unknown words or substituted words with the same beginning sound. On a second study involving 33 eight and nine year old dyslexic children and 31 controls, the results of two of seven cognitive tasks confirmed subtype differences. Significant differences in left temporal parietal theta activity in the electroencephalograms of the dyseidetic children suggested that their reading disabilities may be the result of overuse of linguistic abilities rather than deficient visual spatial skills. (Flynn JM, Deering WM. Subtypes of dyslexia: Investigation of Boder's system using quantitative neurophysiology. Dev Med Child Neur 1989; 31:215-223).

COMENT. A diagnostic screening test for subtypes of reading disability, the Boder Test of Reading Spelling Patterns, is published by the Psychological Corporation, San Antonio, TX. The Boder Test is easily administered and identifies four subtypes of reading disability on the basis of reading and spelling performance. The test is based on the premise that dyslexic readers have characteristic patterns of strengths and weaknesses in two distinct cognitive components of the reading process: The visual gestalt function and the auditory analytic function. The visual gestalt function underlies the ability to develop a sight vocabulary. The auditory analytic function underlies the ability to develop phonic word-analysis skills. These two cognitive functions are basic to the two standard methods of initial reading instruction: The whole 
word method and the phonics method. The Boder test provides a diagnosis that is meaningful to the educator in the choice of remediation methods. This matching method or neuropsychological approach to reading remediation involves matching the learning strengths with a teaching strategy designed to exploit these strengths. It is favored by Johnson and Mykelbust who recognize two main subtypes of dyslexia, "visual dyslexia and auditory dyslexia" and also by Mattis who identified language, dyscoordination, and visuospatial subtypes. The validation of Boder's neuropsychological approach and classification of dyslexic subtypes is an important advance in the evaluation of reading remediation methods. The Boder method, matching learning strengths to teaching methods, appears to be theoretically sound and much preferred to techniques based on deficit remediation which involve the training or retraining of damaged or dysfunctional areas of the brain. (Millichap JG, Millichap NM. Dyslexia: As the neurologist and educator read it. Springfield, IL. Charles C. Thomas, Publisher 1986).

\section{CORPUS CALIOSUM AND OOGNITIVE FUNCTION}

Cognitive functions commicated between the cerebral hemispheres by the corpus callosum was studied in two patients with commissurotamies at the Cognitive Neuroscience Laboratory, Department of Psychiatry, Dartmouth Medical School, Hanover, $\mathrm{NH}$ and the Department of Neurosciences, University of California at San Diego, CA. The patients were asked to judge whether pairs of words rhymed. One word in each pair was presented to the left visual field and the other to the right visual field. The two words in each pair either sounded and looked alike $(R+L+)$, sounded alike but looked different (R+ L-), sounded different but looked alike (R- L+), or both sounded and looked different (R- L-). The two commissurotamy patients differed in that one had sparing of same rostral and splenial fibers of the corpus callosum verified by MRI while the second patient had MRI-verified full callosal section. The patient with some sparing of fibers was able to perform the rhyming judgment significantly better than chance when the words both looked and sounded alike $(\mathrm{R}+\mathrm{L}+)$ whereas her accuracy did not differ from chance in the other three conditions. The second patient with full callosal section performed at chance in all conditions, and normal control subjects were significantly better than chance in all conditions except $\mathrm{R}+\mathrm{L}-$. Both patients had callosal section performed at age 26 to control intractable epileptic seizures. These results indicated to the authors that the first patient was capable of comparing both phonologic and orthographic information across her hemispheres and this ability reflected the functioning of the callosal remant fibers. The splenium of the corpus callosum interconnects visual association cortex and this patient's ability to transfer orthographic information was commensurate with the splenial locus of her spared callosal fibers. The surviving rostral fibers may have contributed to the transfer of phonologic information. (Gazzaniga MS et al. Human callosal function: MRI-verified neuropsychological functions. Neurology July 1989; 39:942-946). 